\title{
Speech valves types and complications, our experience from 521 outpatient visits
}

\section{Mr Hassan Mohammed FRCS (ORL-HNS), Ms Lorna Gamberini, Mr Andrew Robson (FRCS), Cumberland Infirmary}

\section{Introduction:}

Speech valves are the gold standard for voice restoration following laryngectomy. Problems such as leak, granulation and tract loss could affect their use.

We aim to present our experience and an insight to the commonly used speech valves in laryngectomy patients.

\section{Materials and methods:}

Data was collected for patients who had a speech valve over 6 years period. Only patients who underwent laryngectomy with post-operative radiotherapy and fitted with speech valve were included.

\section{Results:}

$57 / 65(87.7 \%)$ of laryngectomy patients in our centre were fitted with speech valves. leak through was the most common reason for valve replacement and hospital visits $309 / 521$ (59.3\%). Granulation was documented in 37 visits and candida in 24. A patient with speech valve would visit the hospital 4 times/year.

\section{Discussion:}

Compared with the literature, the number of speech valve users in our study is higher than what was reported in other studies. With less patients using other methods such as electrolarynx.

Problems with candida were encountered more in Provox 2 and Provox Vega patients in our study cohort. However, it is worth mentioning that even with that, Provox 2 was the valve associated with the least frequent visits when compared with other valves.

Our data showed that Provox 2 (the valve with the largest diameter) was associated with "leak around" in only $1 / 81(1.2 \%)$ of cases while Indwelling, on the other hand, "leak around" was observed in 15/158 (9.5\%) of patients visits. This finding indicates that other factors such as tissue quality after radiotherapy and patient nutritional status could play more important role rather than the size of the valve itself.

\section{Problems encountered in patients with speech valves in their hospital visits}

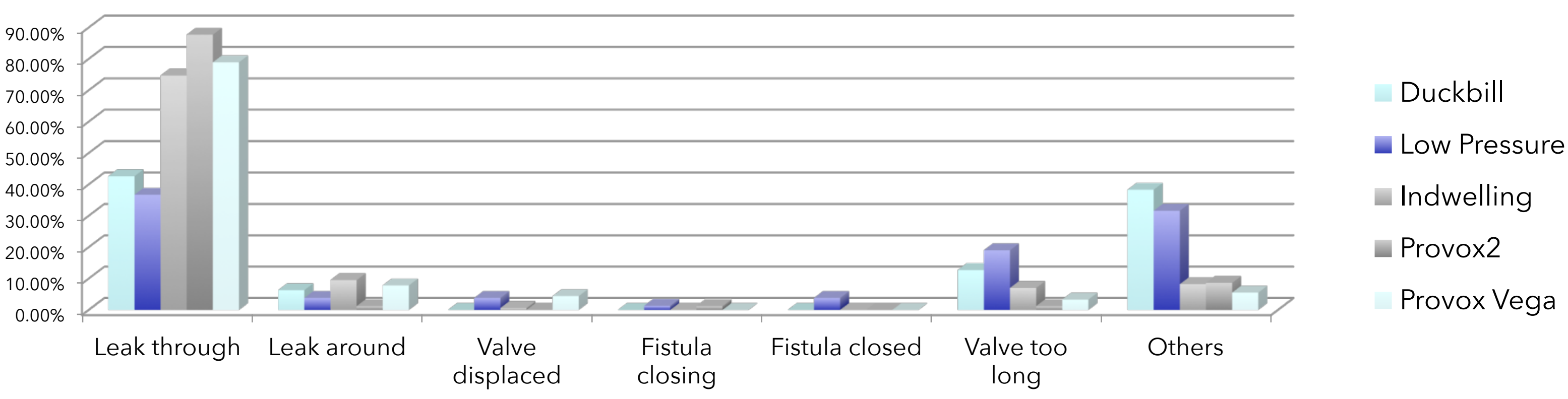

\section{Prevalence of granulation and candida in speech valves}

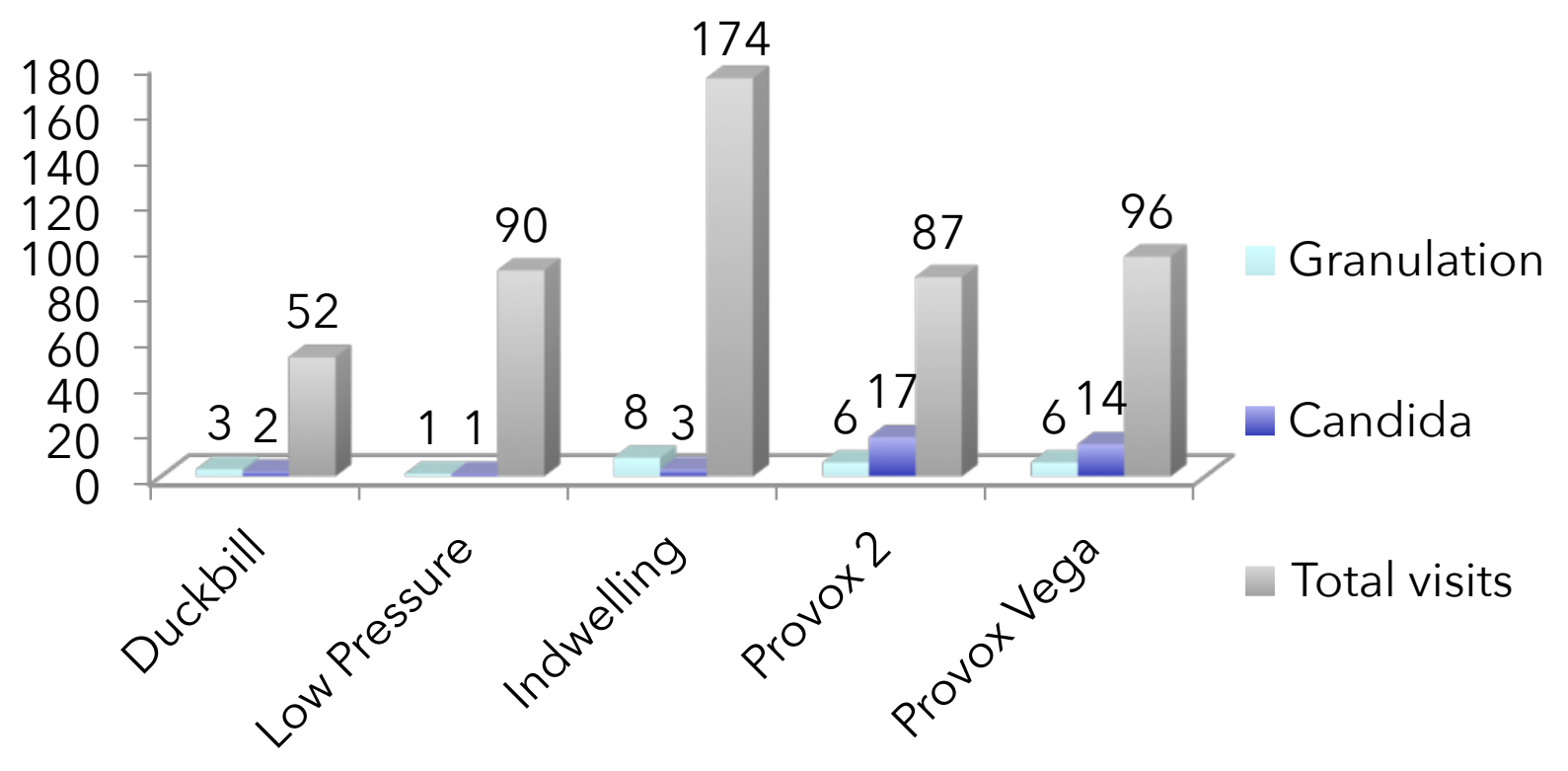

Breakdown of excluded patients

\begin{tabular}{|l|c|}
\hline \multicolumn{1}{|c|}{ Exclusion reason } & Number of patients \\
\hline Recent laryngectomy & 3 \\
\hline Laryngectomy-only & 2 \\
\hline Laryngectomy with post-op CRT & 1 \\
\hline Post-RT laryngectomy & 5 \\
\hline Post-IMRT laryngectomy & 2 \\
\hline Post CRT laryngectomy & 2 \\
\hline Laryngectomy without valve & 8 \\
\hline Total & $\mathbf{2 3}$ \\
\hline
\end{tabular}

\section{Conclusion:}

Based on our experience we recommend that low-pressure valves are good option to start with. It is worth highlighting that low pressure and indwelling valves appear to be the most favorable types in term of lifespan and of encountered problems according to this study. Problems such as granulation and fungal colonization can lead to more frequent valve changes. Further research is encouraged to investigate more effective solutions. 